\title{
High level of telomerase RNA gene expression is associated with chromatin modification, the ALT phenotype and poor prognosis in
} liposarcoma

\author{
CJ Cairney', SF Hoare', M-G Daidone ${ }^{2}$, N Zaffaroni ${ }^{2}$ and WN Keith ${ }^{*, 1}$ \\ 'Centre for Oncology and Applied Pharmacology, University of Glasgow, Cancer Research UK Beatson Laboratories, Garscube Estate, Switchback Road, \\ Bearsden, Glasgow G6I IBD, UK; ${ }^{2}$ Unit 10, Department of Experimental Oncology and Laboratories, Fondazione IRCCS Istituto Nazionale dei Tumori, \\ Via Venezian, I, Milan 20133, Italy
}

\begin{abstract}
Telomere length is maintained by two known mechanisms, activation of telomerase or alternative lengthening of telomeres (ALT). The ALT pathway is more commonly activated in tumours of mesenchymal origin, although the mechanisms involved in the decision of a cell to activate either telomerase or ALT are unknown at present and no molecular markers exist to define the ALT phenotype. We have previously shown an association between chromatin remodelling, telomerase gene expression and ALT in cell line models. Here, we evaluate these findings and investigate their prognostic significance in a panel of liposarcoma tissue samples to understand the biology underlying the ALT phenotype. Liposarcoma samples were split into three groups: telomerase positive (Tel + ); ALT positive; ALT-/Tel-. Differences in telomerase gene expression were evident between the groups with increased expression of $h T R$ in ALT and Tel + compared to ALT-/Tel- samples and increased hTERT in Tel + samples only. Investigation of a small panel of chromatin modifications revealed significantly increased binding of acetyl $\mathrm{H} 3$ in association with hTR expression. We confirm that the presence of the ALT phenotype is associated with poor prognosis and in addition, for the first time, we show a direct association between hTR expression and poor prognosis in liposarcoma patients.

British Journal of Cancer (2008) 98, |467- |474. doi:I0.1038/sj.bjc.6604328 www.bjcancer.com

Published online 15 April 2008

(c) 2008 Cancer Research UK
\end{abstract}

Keywords: chromatin; telomerase; hTR; hTERT; ALT; liposarcoma

The activity of the ribonucleoprotein complex telomerase is reliant on the expression of both hTR and hTERT genes, the regulation of which is tightly coordinated on multiple levels by transcriptional, post-transcriptional (Zhao et al, 2000, 2003, 2005; Cong et al, 2002; Anderson et al, 2006; Bilsland et al, 2006) and epigenetic mechanisms (Atkinson et al, 2005; Serakinci et al, 2006). In most normal somatic cells, telomerase is inactivated following development so that telomeres shorten after each round of replication until they reach a critical length, signalling senescence or cell death. The majority of human tumours reactivate telomerase to maintain telomere length allowing them to bypass senescence and continue to proliferate. However, telomere length maintenance can also be achieved in some tumour types in the absence of telomerase activity through an alternative recombination-based mechanism termed ALT (alternative lengthening of telomeres) (Bryan et al, 1995, 1997). Alternative lengthening of telomeres is characterised phenotypically by heterogeneous telomeres, ranging from less than $3 \mathrm{~kb}$ to more than $50 \mathrm{~kb}$ in length (Henson et al, 2002), and the presence of ALT-associated promyelocytic leukaemia (PML) bodies (APBs), which contain telomeric DNA and the telomere binding proteins, TRF1 and TRF2, in addition to several other proteins associated with DNA recombination and repair,

*Correspondence: Dr WN Keith; E-mail: n.keith@beatson.gla.ac.uk Received 6 December 2007; revised 22 February 2008; accepted 3 March 2008; published online 15 April 2008 including RAD51 and RAD52 (Yeager et al, 1999; Henson et al, 2002).

The ALT pathway is detected in some tumours of epithelial origin, including carcinomas of the breast, lung and kidney; however, it is found at higher frequency in tumours of mesenchymal origin, such as liposarcomas, osteosarcomas and glioblastomas (Henson et al, 2002). This is thought to be due to tighter repression of telomerase expression in mesenchymal tissues, which have a slower cell turnover and less telomere shortening than many epithelial tissues (Henson et al, 2002). Several studies have highlighted an association of the ALT phenotype with sarcomas that have complex karyotypes with many chromosomal losses and gains and evidence of anaphase bridge formation, more consistent with chromosomal instability, while those that have generally more simple karyotypes, including fusion genes created from chromosomal translocations, are associated with telomerase activity (Montgomery et al, 2004; Ulaner et al, 2004).

Liposarcomas are among the most common soft tissue sarcomas forming around $20 \%$ of all mesenchymal tumours (Sandberg, 2004). They are a diverse group of mesenchymal malignancies that show multiple telomere maintenance mechanisms (TMMs) with telomerase activity and the ALT phenotype detected in a similar number of cases (Johnson et al, 2005; Costa et al, 2006). Alternative lengthening of telomeres is more frequent in welldifferentiated and dedifferentiated forms (Costa et al, 2006), which 
show complex karyotypic rearrangements including supernumerary ring, giant chromosome formation and amplification (Sandberg, 2004), while telomerase activity is more frequent in myxoid forms (Costa et al, 2006) characterised by the $\mathrm{t}(12 ; 16)(\mathrm{q} 13 ; \mathrm{p} 11)$ translocation (Sandberg, 2004). In addition, a significant proportion appears to show no evidence of any known TMM (Johnson et al, 2005; Costa et al, 2006). This complexity makes liposarcoma a good model system to investigate the regulation of TMMs.

The presence of any TMM, in comparison to tumours that express neither telomerase nor ALT, is associated with poor prognosis in a number of tumours of mesenchymal origin (Sanders et al, 2004; Costa et al, 2006). In osteosarcoma, telomerase expression is associated with shorter progression-free survival (Sanders et al, 2004), while in liposarcoma the ALT phenotype is associated with a lower survival rate (Costa et al, 2006). This appears to be tissue specific, as the presence of the ALT phenotype in patients with glioblastoma multiforme was associated with a longer survival rate than those with telomerase activity or no TMM (Hakin-Smith et al, 2003).

The molecular mechanisms involved in the decision to activate either telomerase or the ALT pathway within a particular cell type are so far unknown. As mentioned above, the presence of APBs is a defining characteristic of the ALT phenotype. A recent study showed the requirement of PML, TRF1, TRF2, TIN2, RAP1 and the MRN complex proteins MRE11, RAD50 and NBS1 for APB formation in ALT cells. The close association of APB formation with ALT highlights these genes as candidate markers or regulators of the ALT pathway (Jiang et al, 2007); however, the mechanisms of regulation remain to be fully determined. We have recently shown that chromatin remodelling at the telomerase gene promoters is associated with a lack of expression of hTR and hTERT in some ALT cell lines, highlighting one potential mechanism for regulating the activation of ALT or telomerase (Atkinson et al, 2005). Here, we analyse telomerase gene expression and the association of a small panel of chromatin modifications, with expression of these genes in liposarcoma samples with relation to TMM and patient prognosis, to investigate the molecular regulation of the ALT phenotype in tumour tissue. The identification of molecular regulators or markers of the ALT pathway will be useful not only as prognostic indicators of patient survival but may also highlight areas for therapeutic intervention to treat these tumours more effectively.

\section{MATERIALS AND METHODS}

\section{Study population}

Samples from 27 liposarcomas, all from adult patients treated with curative intent at the Istituto Nazionale Tumori of Milan from December 1986 to November 2003 were available for TMM, telomerase gene expression and chromatin modification analyses. These specimens represent a subset randomly selected from a larger case series already characterised for TMM (Costa et al, 2006). There were 13 women and 14 men, with a median age of 58 years (range, 38-91). Four patients presented at Istituto Nazionale Tumori of Milan with primary disease and 23 with recurrent tumours (22 recurrences and 1 metastasis), and they underwent different surgical procedures according to disease presentation. Tumour characteristics in terms of histology and grade are reported in Table 1. Postoperative treatment was given when there were clinical grounds for concluding that a high risk of recurrence existed: eight patients were submitted to radiotherapy, two to chemotherapy, and one to radiochemotherapy according to the treatment protocols of the multidisciplinary Istituto Nazionale Tumori of Milan Soft Tissue Sarcoma Group. The median follow-up for the entire group as of December 2006 was 108 months. During the follow-up period, 12 patients died of liposarcoma-related
Table I Main tumour characteristics

\begin{tabular}{|c|c|c|c|c|c|}
\hline Tumour & Grade & $\begin{array}{l}\text { Histological } \\
\text { subtype }\end{array}$ & TMM & $\begin{array}{l}\text { hTR } \\
\text { expression }\end{array}$ & $\begin{array}{l}\text { hTERT } \\
\text { wild-type } \\
\text { expression }\end{array}$ \\
\hline SI & I & WD & ALT-Tel- & Low & No \\
\hline $\mathrm{S} 2$ & I & WD & ALT-Tel- & Low & No \\
\hline S3 & 2 & WD & ALT-Tel- & High & No \\
\hline S4 & I & WD & ALT-Tel- & High & No \\
\hline S5 & I & WD & ALT-Tel- & High & No \\
\hline S6 & 2 & WD & ALT-Tel- & Low & No \\
\hline S7 & 1 & Myx & ALT-Tel- & High & No \\
\hline S8 & 1 & WD & ALT-Tel- & Low & No \\
\hline S9 & 1 & WD & ALT-Tel- & Low & No \\
\hline SIO & 1 & DD & $\mathrm{ALT}+$ & No result & No \\
\hline SII & 1 & DD & $\mathrm{ALT}+$ & High & No \\
\hline $\mathrm{S} 12$ & 3 & DD & $\mathrm{ALT}+$ & High & No \\
\hline $\mathrm{S} 13$ & 3 & DD & $\mathrm{ALT}+$ & High & No \\
\hline $\mathrm{S} 14$ & 3 & $\mathrm{RC}$ & $\mathrm{ALT}+$ & High & No \\
\hline SI5 & 2 & DD & $\mathrm{ALT}+$ & Low & Yes \\
\hline S16 & 3 & DD & $\mathrm{ALT}+$ & High & No \\
\hline $\mathrm{S} 17$ & 3 & DD & $\mathrm{ALT}+$ & High & No \\
\hline $\mathrm{S} 18$ & 2 & Myx & $\mathrm{ALT}+$ & Low & No \\
\hline S19 & 1 & $\mathrm{RC}$ & $\mathrm{ALT}+$ & High & No \\
\hline $\mathrm{S} 20$ & 2 & Myx & Tel+ & High & Yes \\
\hline $\mathrm{S} 21$ & 3 & Myx & Tel+ & High & Yes \\
\hline $\mathrm{S} 22$ & 2 & DD & Tel+ & High & No \\
\hline $\mathrm{S} 23$ & 1 & $\mathrm{RC}$ & Tel+ & Low & No \\
\hline S24 & 2 & $\mathrm{RC}$ & Tel+ & High & Yes \\
\hline $\mathrm{S} 25$ & 2 & DD & Tel+ & High & Yes \\
\hline $\mathrm{S} 26$ & I & Myx & Tel+ & High & Yes \\
\hline S27 & 2 & $\mathrm{RC}$ & Tel+ & High & Yes \\
\hline
\end{tabular}

$\mathrm{DD}=$ dedifferentiated; $M y x=$ usual myxoid; $\mathrm{RC}=$ round-cell myxoid; $\mathrm{WD}=$ well differentiated. Cutoff for low or high hTR expression was taken as the mean of all samples minus two standard errors.

causes, 5 were alive with disease and 7 were disease free. Three patients were lost to follow-up.

All patients provided written informed consent to donate the tissue that was left over after diagnostic procedures were completed to the Istituto Nazionale Tumori of Milan.

\section{Detection of APBs and telomerase activity}

Tumour tissue was sampled by a pathologist at the time of surgery and fresh-frozen. Detection of APBs by combined PML immunofluorescence and telomere fluorescence in situ hybridisation (FISH) and telomerase activity by the telomeric-repeat amplification protocol (Kim et al, 1994) with the TRAPeze kit (Intergen Company, Oxford, UK) were carried out as outlined in Costa et al (2006).

\section{Sample preparation for RNA extraction and ChIP assay}

Frozen tissue samples varying in size from 50 to $120 \mathrm{mg}$ were disrupted into small pieces with liquid nitrogen and a mortar and pestle and then split in half for use in chromatin immunoprecipitation (ChIP) assay and RNA extraction for telomerase gene expression analysis.

\section{Expression analysis}

Further disruption of the tissue was carried out in RA1 buffer plus $\beta$-mercaptoethanol (Nucleospin II RNA extraction kit, MachereyNagel, Düren, Germany) in a Ribolyser (Hybaid, Waltham, MA, USA) at a setting of 5.5 for $5 \times 10 \mathrm{~s}$ pulses with $30 \mathrm{~s}$ pauses between. Samples were then passed through an 18-gauge needle to shear genomic DNA. RNA was extracted using the Nucleospin II RNA extraction kit (Macherey-Nagel) following the manufacturer's 
instructions. hTR expression was analysed by quantitative PCR (Q-PCR) as described previously (Atkinson et al, 2005) and taken as a percentage of the riboprotein S15 (primers (RETROscript kit, Ambion) S15 Forward TTCCGCAAGTTCACCTACC, S15 Reverse CGGGCCGGCCATGCTTTACG). hTERT expression was analysed by semiQ-PCR using primers designed to detect the four main hTERT splice variants (Atkinson et al, 2005). Samples were adjusted for S15 expression prior to splice variant PCR, and total hTERT levels were taken as the sum of all splice variant products quantified using the Agilent Bioanalyser as described previously (Keith and Hoare, 2004).

\section{Chromatin immunoprecipitation}

Chromatin immunoprecipitation assays were carried out using the Upstate Biotechnologies protocol as described previously (Atkinson et al, 2005), with some modifications for tissue handling adapted from Farnham's ChIP protocol for tissues (http:// genomics.ucdavis. edu/farnham/). Half of the disrupted tumour sample was fixed in PBS containing 1\% formaldehyde. After $10 \mathrm{~min}, 0.125 \mathrm{M}$ glycine was added to quench the formaldehyde and stop the cross-linking. Tissue was rinsed in cold PBS and further disrupted in a dounce homogeniser. Cells were lysed in Farnham's cell lysis buffer ( $5 \mathrm{~mm}$ PIPES, pH 8.0; $85 \mathrm{~mm} \mathrm{KCl;} \mathrm{0.5 \%} \mathrm{NP40;} 1 \mathrm{~mm}$ PMSF; $2 \mu \mathrm{g} \mathrm{ml}^{-1}$ aprotonin; $1 \mu \mathrm{g} \mathrm{ml}^{-1}$ pepstatin) using $500 \mu \mathrm{l}$ per $50 \mathrm{mg}$ tissue, then passed through a series of needles (18-25 gauge) to release the nuclei. Samples were resuspended in SDS lysis buffer (Upstate Biotechnologies, Watford, UK) with protease inhibitors ( $400 \mu \mathrm{l}$ buffer per $50 \mathrm{mg}$ tissue) and incubated on ice for $90 \mathrm{~min}$. The remainder of the protocol was carried out as described (Atkinson et al, 2005); however, no DNA purification was required.

\section{Quantitative PCR}

Products from ChIP assays were analysed by Q-PCR as described previously (Atkinson et al, 2005). Data are presented as a percentage of input following subtraction of the background pulled down with the no antibody control.

\section{Antibodies}

Antibodies used were AcH3, AcH4 and TriMeK20 (all Upstate Biotechnologies) and TriMeK9 (Abcam, Cambridge, UK). Tissue (13-25 mg) was required per antibody.

\section{Data analysis}

Criteria used to define the presence of telomerase and ALT phenoypes were previously detailed (Costa et al, 2006). The cutoff to discriminate between low and high hTR expression was defined as $97 \%$ of S15 expression (mean of all samples minus two standard errors). hTERT-positive cases were defined as those expressing the wild-type variant. In vitro measurements were performed by personnel blinded to patient data and clinical outcome, whereas clinical data were collected by personnel blinded to in vitro data. Kruskal-Wallis, Mann-Whitney and $\chi^{2}$ tests were used to assess differences in the distribution of data categorised according to the investigated features. The clinical end point of this study was cause-specific mortality, and the time of its occurrence was computed from the date of first diagnosis to the time of death or censored at the date of the last recorded follow-up for living patients or for those who died from liposarcoma-unrelated conditions. Survival analysis was carried out with Cox regression models after checking for the proportional hazard assumption. SAS software (SAS Institutes Inc., Cary, NC, USA) was used for statistical calculations. All $P$-values were two-sided; $P<0.05$ was considered to be statistically significant.

\section{RESULTS}

\section{How does telomerase gene expression relate to the ALT phenotype?}

A total of 27 liposarcoma tissue samples were collected from 27 different patients and evaluated for telomerase activity using the TRAP assay and for the ALT phenotype using immunofluorescence/FISH to detect ALT-associated PML bodies. The samples were then split into three groups depending on TMM status: telomerase positive $(\mathrm{Tel}+)$; ALT positive; both Tel-/ALT-. To explore the molecular mechanisms underlying the morphological determination of the ALT phenotype, we analysed expression of hTR and hTERT at the RNA level.

hTR expression, normalised to expression of the riboprotein S15, was determined by Q-PCR and expressed as a percentage of S15 (Figure 1A). All of the liposarcoma samples evaluated in this study expressed hTR to varying degrees; however, significant differences in expression were found within the three groups $(P=0.05)$. Samples that were negative for both telomerase and ALT had mostly low-level expression with a median level of $48 \%$ of S15 expression (range 20-333\%), while those that were Tel + had an even distribution of expression with a median level of $242 \%$ (range $31-562 \%$ with one outlier at $1783 \%$ ). Those samples expressing the ALT phenotype, on the other hand, appeared to have a higher distribution of expression, although the median value of $199 \%$ did not reflect this (range $71-653 \%$ with one outlier at $3575 \%$ ).

hTERT expression evaluated by semi-Q-PCR is shown in Figure 1B. The majority of liposarcoma samples, which were negative for both telomerase and ALT or expressed the ALT phenotype, had little or no total hTERT expression. The median level for both of these groups was $0 \mathrm{ng} \mu \mathrm{l}^{-1}$. Conversely, the majority of samples that were Tel + expressed significantly higher levels of hTERT in comparison to the other groups, with a median level of $9.4 \mathrm{ng} \mathrm{l}^{-1}$. TERT expression is known to be regulated by differential splicing; therefore, we investigated the expression pattern of the four main splice variants of hTERT (Figure 1C). Little expression of any of the splice variants was detected in the samples, which were negative for both telomerase and ALT. The inactive beta variant was detected in two of these samples and the active wild-type variant was not detected at all. Likewise, for samples expressing the ALT phenotype, three samples expressed the inactive beta variant and only one sample expressed the active wild-type variant. It is of interest to note that this sample is the only one in which the dominant-negative alpha variant is detected and it is one of only two ALT samples expressing no hTR. In contrast, the active wild-type variant could be detected in the majority of the Tel + samples with the exception of samples 22 and 23. Lack of the wild-type variant in these samples may reflect differences in sensitivity between the TRAP assay and the splice variant PCR.

Table 2 provides a summary of the expression data generated for the liposarcoma samples separated by phenotype and expression, where hTR + is taken as over $97 \%$ of S15 expression (mean of all samples minus two standard errors) and hTERT + is taken as expressing the wild-type splice variant. A statistically significant $(P=0.004)$ association was observed between the absence or presence of TMMs and telomerase gene expression. Telomerase enzymatic activity is dependent on expression of both hTR and hTERT; therefore, it is interesting to note that the only samples positive for both hTR and hTERT expression lie within the Tel+ group. The fact that two other samples lie within the Tel + group, although they appear to be negative for hTERT or both hTERT and hTR, could reflect differences in assay sensitivity rather than phenotype, as mentioned previously. The majority of samples expressing the ALT phenotype, on the other hand, express only hTR, with the exception of one sample that expresses hTERT alone 
A
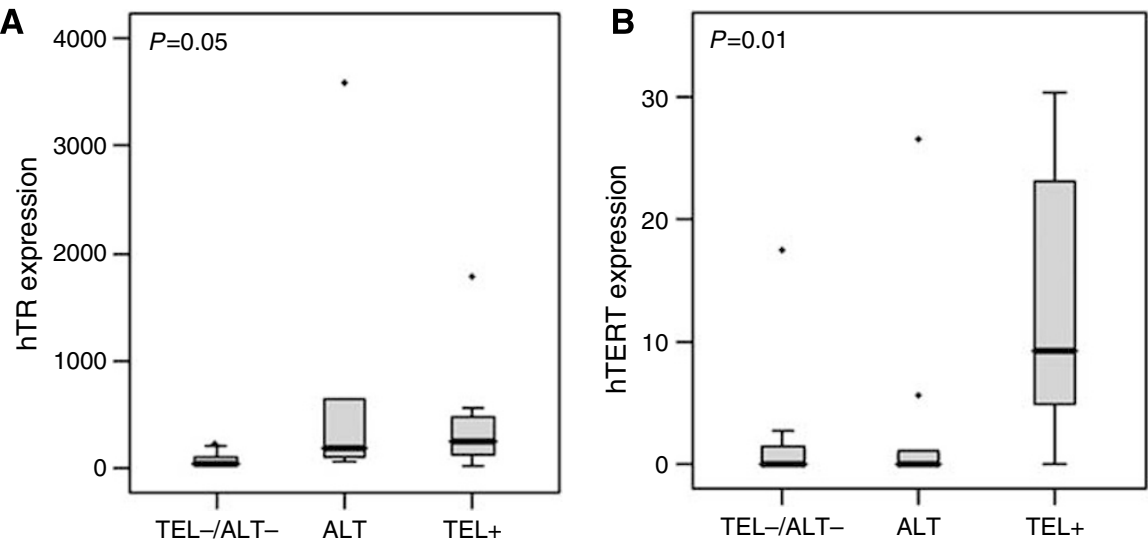

C

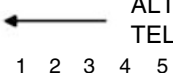

TEL- $\longrightarrow$ ALT

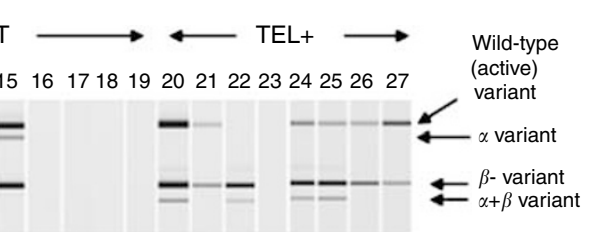

Figure I Telomerase gene expression in liposarcoma samples. (A) hTR expression taken as a percentage of the riboprotein SI5; (B) total hTERT expression in $\mathrm{ng}$ per $\mu \mathrm{l}$. The grey box defines 25 and 75\% quartiles, while error bars represent the first and 99th percentiles of the distribution. Dots represent outliers and the black line defines the median of the distribution. P-values were calculated using the Kruskal-Wallis test, which tests the likelihood of all medians being the same. (C) Four major hTERT splice variant PCR products were quantified using the Agilent Bioanalyser.

Table 2 Summary of telomerase gene expression in relation to ALT phenotype or telomerase status

\begin{tabular}{lcccc}
\hline & $\begin{array}{c}\text { hTR-I } \\
\text { hTERT- }\end{array}$ & $\begin{array}{c}\text { hTR-I } \\
\text { hTERT+ }\end{array}$ & $\begin{array}{c}\text { hTR+/ } \\
\text { hTERT- }\end{array}$ & $\begin{array}{c}\text { hTR+/ } \\
\text { hTERT+ }\end{array}$ \\
\hline ALT-l & 5 & 0 & 4 & 0 \\
TEL- & 2 & 1 & 7 & 0 \\
ALT+ & 1 & 0 & 1 & 6 \\
TEL+ & 1 & & & \\
\hline
\end{tabular}

and two more that have neither hTERT nor hTR expression. A high level of hTR and little or no hTERT expression in association with the ALT phenotype has been shown previously in cell line models (Hoare et al, 2001; Atkinson et al, 2005). Finally, samples lacking both telomerase expression and ALT are negative for hTR and hTERT or express only hTR.

\section{How does the chromatin landscape relate to telomerase gene expression in liposarcoma?}

It is well known that gene expression can be controlled at the level of chromatin and we have previously shown that telomerase gene expression is subject to epigenetic regulation by chromatin remodelling in cell line models. To determine whether modification of the chromatin landscape bears any relation to telomerase gene expression in liposarcoma, we used chromatin immunoprecipitation to study the association of a small panel of histone modifications with the telomerase gene promoters. Samples were grouped according to levels of hTR expression or by the presence or absence of the hTERT wild-type splice variant. The association of two modifications related to active gene expression, acetyl histone $\mathrm{H} 3$ and acetyl histone $\mathrm{H} 4$, and two modifications related to gene repression and the heterochromatic state, tri-methyl lysine 9 of histone $\mathrm{H} 3$ and tri-methyl lysine 20 of histone H4, with the hTR and hTERT promoters were investigated within these expression groups (Figures 2 and 3, respectively).
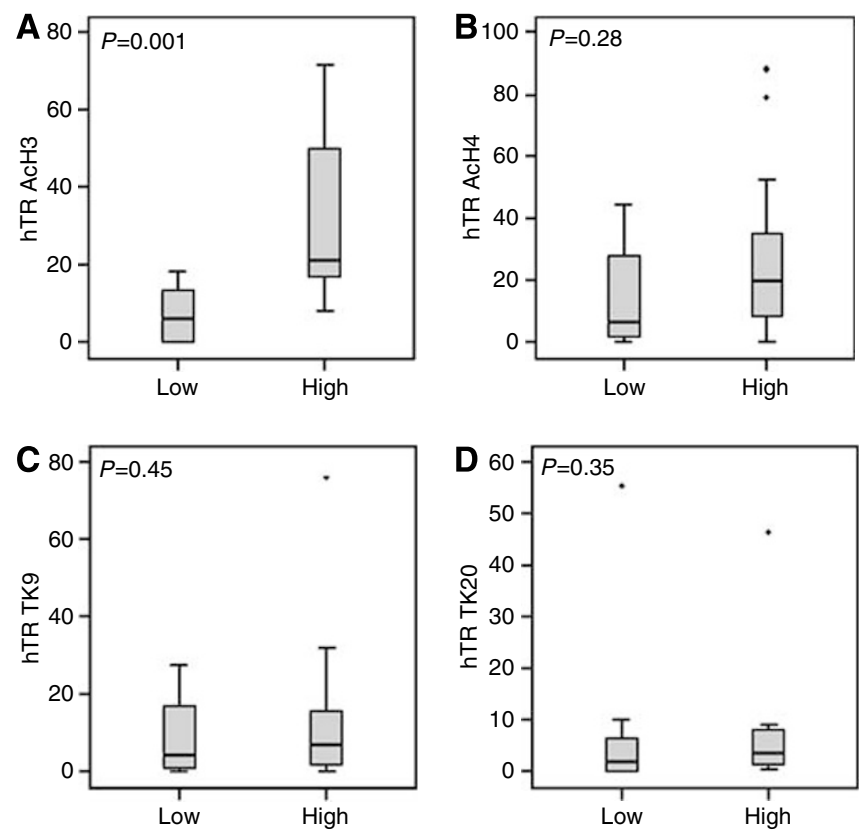

Figure 2 Chromatin modifications associated with low and high hTR expressing liposarcoma samples represented as a percentage of input chromatin. (A) Association of acetyl histone H3; (B) association of acetyl histone $\mathrm{H} 4$; (C) association of tri-methyl $\mathrm{K} 9$ of H3; (D) association of trimethyl $\mathrm{K} 20$ of $\mathrm{H} 4$. The grey box defines 25 and $75 \%$ quartiles, while error bars represent the first and 99th percentiles of the distribution. Dots represent outliers and the black line defines the median of the distribution. $P$-values were calculated using the Mann-Whitney test, which tests the likelihood of all medians being the same.

The data in Figure 2 show a relationship between hTR expression and acetylation of histones $\mathrm{H} 3$ and $\mathrm{H} 4$. Association of acetyl H3 (Figure 2A) with the hTR promoter is significantly 

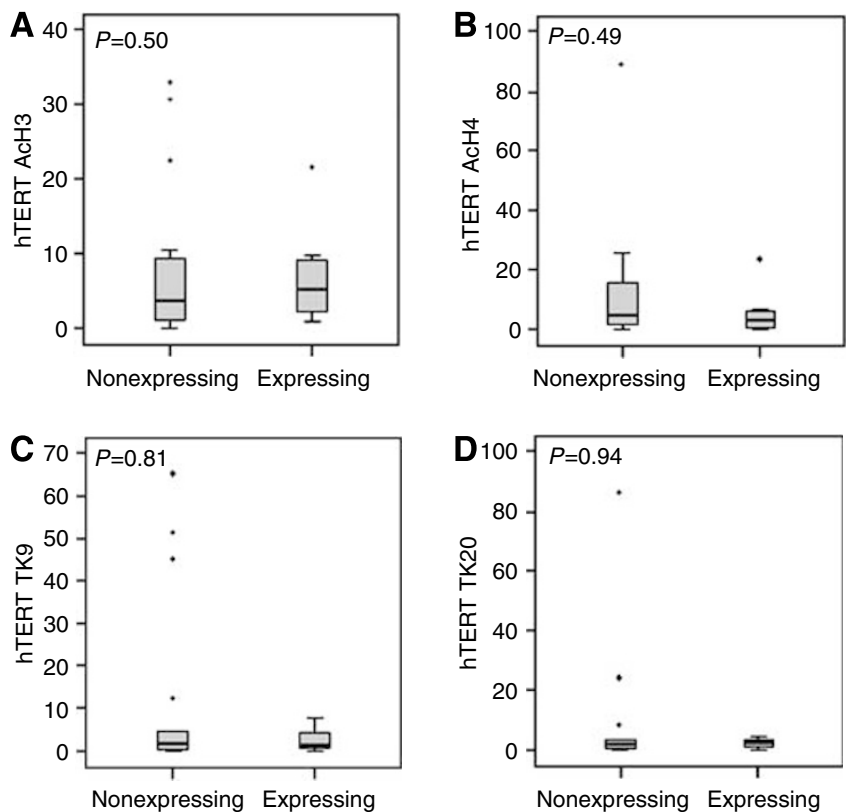

Figure 3 Chromatin modifications associated with liposarcoma samples expressing wild-type hTERT or not represented as a percentage of input chromatin. (A) Association of acetyl histone H3; (B) association of acetyl histone $\mathrm{H} 4$; (C) association of tri-methyl K9 of H3; (D) association of trimethyl K20 of $\mathrm{H} 4$. The grey box defines 25 and $75 \%$ quartiles, while error bars represent the first and 99th percentiles of the distribution. Dots represent outliers and the black line defines the median of the distribution. $P$-values were calculated using the Mann-Whitney test, which tests the likelihood of all medians being the same.

increased as a function of hTR expression (median levels of 5.5 and $20.8 \%$ of input for low and high expressors, respectively; $P=0.001$ ), while the increase in association of acetyl $\mathrm{H} 4$ with the hTR promoter in high expressors compared to low expressors (median levels of 19.4 and $6.3 \%$ of input, respectively) did not reach significance $(P=0.28)$. In the case of the negative modifications, no significant differences in association were seen between high and low expressing samples (Figure $2 \mathrm{C}$ and $\mathrm{D}$ ). These results suggest that hTR expression in these liposarcoma samples is associated with positively acting histone modifications and that the negative modifications we have chosen for this study bear no relation to hTR gene expression.

The relationship between binding of these chromatin modifications and hTERT expression is less obvious, as there are no significant differences between those samples expressing the wildtype variant and those that do not (Figure 3). Although the small differences that we do see in association of these modifications appear uninformative and are not statistically significant, the actual levels of hTERT expression are very low with normal levels of 1-30 copies of the gene per cell (Yi et al, 2001); therefore, these slight differences in chromatin occupancy could be biologically significant. It could be that for hTERT the lack of negatively acting chromatin modifications is more important than the presence of positive modifications, at least of those chosen for this study.

\section{Does chromatin modification at the telomerase gene promoters distinguish between the differential expression of the three phenotypes?}

The results of the previous section suggest that while acetylation of histones at the hTR promoter was related to hTR expression levels, no clear pattern of regulation could be seen for hTERT. To further study the mechanism of regulation of the TMM phenotype, we next looked at the potential of these chromatin modifications as
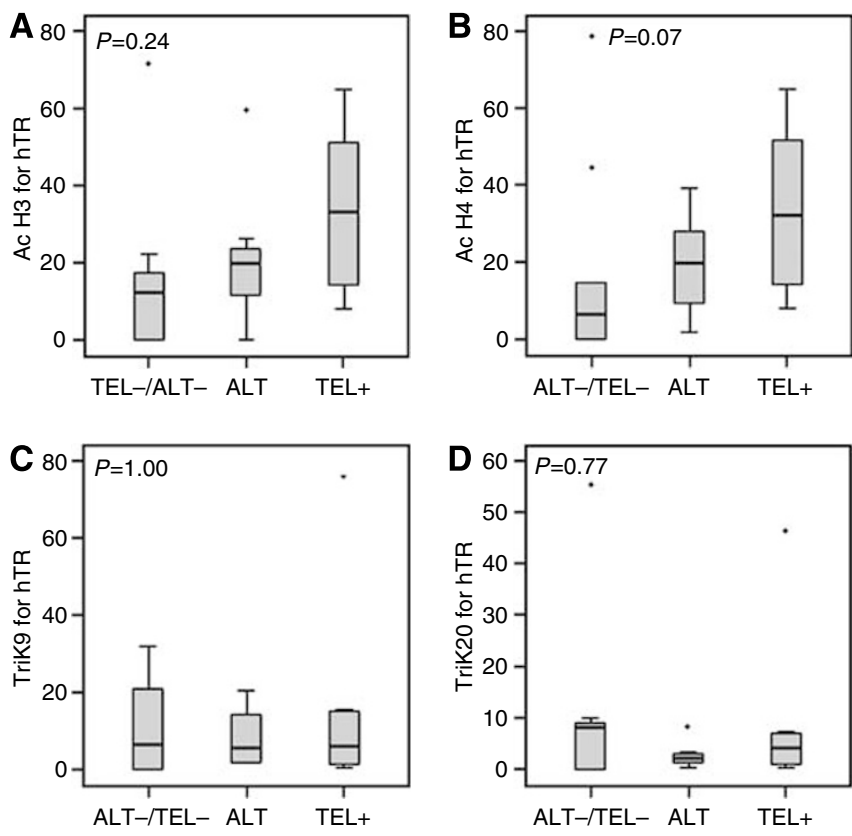

Figure 4 Chromatin modifications at the hTR promoter in samples grouped by phenotype represented as a percentage of input chromatin. (A) Association of acetyl histone H3; (B) association of acetyl histone $\mathrm{H} 4$; (C) association of tri-methyl K9 of H3; (D) association of tri-methyl K20 of H4. The grey box defines 25 and $75 \%$ quartiles, while error bars represent the first and 99th percentiles of the distribution. Dots represent outliers and the black line defines the median of the distribution. P-values were calculated using the Kruskal-Wallis test, which tests the likelihood of all medians being the same.

molecular markers to distinguish among the three phenotype groups within our liposarcoma samples. Samples were grouped according to telomerase status or ALT phenotype following chromatin immunoprecipitation as previously.

Figure 4 shows association of chromatin modifications at the hTR promoter within the three phenotype groups. Although none of the differences in median levels reached statistical significance, clear trends were seen in the association of positively and negatively acting chromatin modifications across the three phenotype groups. Consistent with expected results, the highest levels of the positively acting marks, acetylation of $\mathrm{H} 3$ (Figure 4A) and H4 (Figure 4B), were found in Tel + samples (median levels of 32.3 and $23.7 \%$ of input, respectively), which also have the highest median levels of hTR expression. No clear association was observed between the levels of the negatively acting marks, trimethyl K9 (Figure 4C) and tri-methyl K20 (Figure 4D) and the distinct TMM subsets. As was the case with expression levels for hTR, levels of acetylation in those samples expressing the ALT phenotype lie somewhere between those those were either positive for telomerase or negative for both telomerase and ALT (Figure 4A and $\mathrm{B}$ ).

In agreement with hTERT expression, the results for association with the hTERT promoter are not so clear and no obvious relationships between binding of the chosen chromatin modifications and telomerase status or ALT phenotype were found (data not shown).

\section{How do telomerase gene expression and the ALT phenotype relate to clinical outcome in patients with liposarcoma?}

To determine whether telomerase gene expression and the ALT phenotype would bear any relationship to patient survival, the clinical outcome was evaluated at the median time of follow-up, 


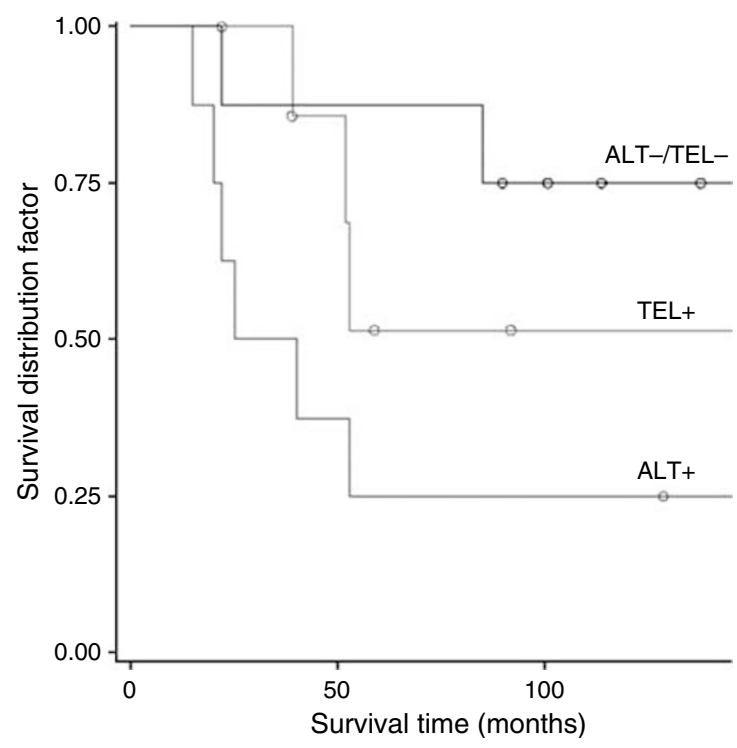

Figure 5 Overall survival as a function of TMM. Comparison between patients with ALT-/Tel- tumours and those with telomerase-positive or ALT tumours.

which in the present series was 9 years. The presence or absence of TMMs segregated liposarcoma patients into distinct prognostic subsets (Figure 5): in fact, 9-year probability of survival was $75 \%$ for the 9 patients with ALT-/Tel- tumours, $51 \%$ for the 8 patients with telomerase-positive tumours and only $25 \%$ for the 10 patients with ALT-positive tumours. Specifically, compared to patients with ALT-positive tumours, those with ALT-/Tel- or with telomerasepositive tumours had a 0.19 -fold $(95 \%$ confidence limit (CL), $0.04-0.94, P=0.042)$ and a 0.38 -fold $(95 \% \mathrm{CL}, 0.10-1.53, P=0.17)$ hazard of death. However, when adjusted for tumour grade, the lower hazard of death for patients with ALT-/Tel- tumours vs those with ALT-positive tumours became only suggestive of statistical significance (hazard ratio (HR), 0.15; 95\% CL, 0.016 $1.48, P=0.09$ ).

Tumour grade and the histological subtype of the liposarcoma samples are important considerations that should be taken into account when looking at the relationship between telomerase gene expression, TMM and clinical outcome. Consistent with previous results (Costa et al, 2006), clinical outcome and TMM correlate with histological subtype. Those in ALT or Tel-positive groups, which have a poorer prognosis, have an increased incidence of the more aggressive liposarcoma subtypes, dedifferentiated or usual myxoid and round-cell myxoid subtypes, respectively, while the well-differentiated tumours were the most abundant subtype within the ALT-/Tel- group, which has the best prognosis (summarised in Table 3). Similarly, taking into account tumour grade, a statistically significant association $\left(P=0.016 ; \chi^{2}\right.$ test $)$ was observed between TMM and grade. Grade 1 tumours were mainly represented by ALT-/Tel- cases (7/12), grade 2 by Tel-positive (5/9) and grade 3 by ALT-positive (5/6) (reported in Table 1).

The results of this study suggest that levels of telomerase gene expression can distinguish among the three TMM phenotype groups; therefore, the relationship of hTR and hTERT expression to prognosis was considered in two ways, by taking the liposarcoma samples as one whole group or by subdividing this large group by the presence or absence of the ALT phenotype. Firstly, we looked at the prognostic significance of hTR and hTERT in all liposarcoma samples. When considered as dichotomous variables, using the mean of all samples minus two standard errors (97\% of S15 expression), as a cutoff to define low or high hTR expression, or any detectable level of wild-type variant for hTERT, no association was observed between these two variables and
Table 3 Summary of histological subtype in relation to ALT phenotype or telomerase status and hTR expression

\begin{tabular}{lcccc}
\hline & $\begin{array}{c}\text { Well } \\
\text { differentiated }\end{array}$ & $\begin{array}{c}\text { Usual } \\
\text { myxoid }\end{array}$ & $\begin{array}{c}\text { Round-cell } \\
\text { myxoid }\end{array}$ & Dedifferentiated \\
\hline ALT-1 & 8 & 1 & 0 & 0 \\
Tel- & 0 & 1 & 2 & 7 \\
ALT+ & 0 & 3 & 3 & 2 \\
Tel+ & 0 & 212 & 271 & 393 \\
hTR \% & 48 & $(96-562)$ & $(31-653)$ & $(7 \mid-3575)$ \\
SI5 & $(20-232)$ & & & \\
\hline
\end{tabular}

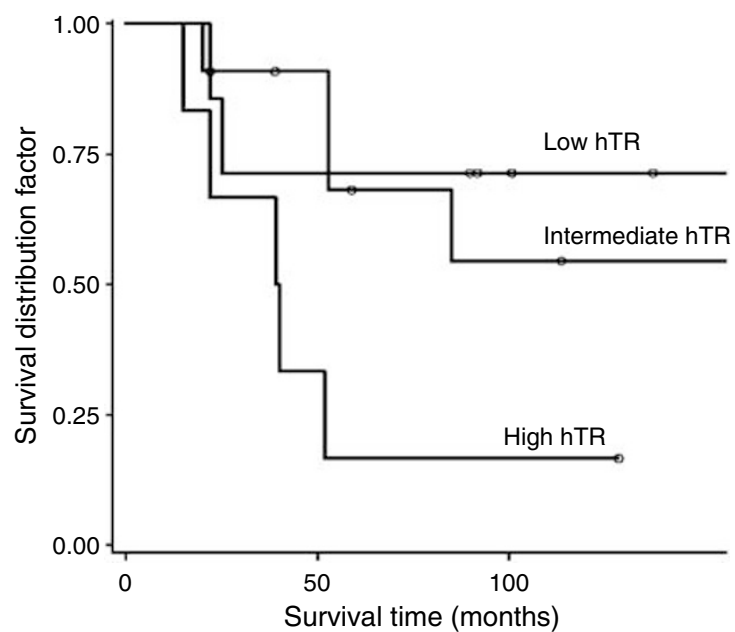

Figure 6 Overall survival as a function hTR expression. Comparison among patients with tumours expressing low, intermediate and high hTR levels. High level of telomerase RNA classes were defined on the basis of its frequency distribution, using the first and the third quartiles as cutoff values.

survival (HR and 95\% CLs for hTR, 1.65 and 0.44-6.24, $P=0.46$ HR and 95\% CL for hTERT, 1.57 and 0.45-5.47, $P=0.48$ ). Conversely, when hTR abundance was considered as a continuous variable, a trend in favour of a worse prognosis for patients whose tumours expressed the higher levels was observed $(P=0.067$, Figure 6), which reached statistical significance after logarithmic transformation (HR and 95\% CL, 1.65 and 1.03-2.65, $P=0.036$ ). In contrast, hTERT expression failed to provide prognostic information, even when considered as a continuous variable.

We next looked at the prognostic significance of hTR and hTERT adjusted for the most important variable in this series, the presence or absence of ALT. While hTERT expression gave no prognostic information, hTR expression had some prognostic significance within ALT- (HR and 95\% CL, 2.15 and 0.97-6.52, $P=0.058)$, but not within ALT + subsets.

The association between high hTR expression and poor prognosis led us to investigate any relationship between hTR expression and histological subtype or tumour grade. When considered as a dichotomous variable, using the mean of all samples minus two standard errors as the cutoff, no significant association was shown between hTR expression and histological subtype or tumour grade of the liposarcoma samples. However, when the median expression levels were considered, we found a trend in association, with the lowest hTR expression in those tumours of the well-differentiated subtype compared to the other more aggressive subtypes (summarised in Table 3 ). Similarly, a significantly higher hTR expression $(P=0.0136$; Kruskal-Wallis test) was observed in grade 3 tumours (median value, 601) compared to grade 2 (median value, 116) or grade 1 tumours (median value, 100). 
Overall, from these data, it would appear that the presence of the ALT phenotype and high levels of hTR expression are singly associated with poor prognosis in liposarcoma patients.

\section{DISCUSSION}

In this study, we sought to investigate the mechanisms regulating the ALT phenotype in liposarcoma tissue samples, with specific emphasis on telomerase gene regulation, to determine how the morphological definition of ALT relates to the underlying biology and clinical outcome. Significant differences in the expression of hTR were found among the three phenotype groups, with samples positive for telomerase or ALT having higher expression levels than those that were negative for both telomerase and ALT. Expression of hTERT, on the other hand, was significantly increased only in Tel + samples.

Liposarcoma samples were initially separated according to their TMM using telomerase activity and the presence or absence of APBs. At present, we have little indication of how these TMMs are regulated at the molecular level to determine which is activated in particular tumour types. We have previously shown in cell line models that regulation of telomerase gene expression is subject to control at the epigenetic level. Methylation of $\mathrm{CpG}$ islands within the hTR promoter in several ALT cell lines was associated with gene repression (Hoare et al, 2001), while remodelling of the chromatin environment in the promoters of both hTR and hTERT was associated with differential gene expression in Tel + or ALT positive tumour cell lines and normal cell line controls (Atkinson et $a l, 2005)$ and repression in human mesenchymal stem cells (Serakinci et al, 2006). Furthermore, treatment of Tel-cell lines with the histone deacetylase inhibitor Trichostatin A and the demethylating agent 5-aza 2 'deoxycytidine induced expression of hTR and hTERT, consistent with decreased methylation and increased association of acetylated histones at these gene promoters (Atkinson et al, 2005; Serakinci et al, 2006). To evaluate these cell line data in clinical samples, we investigated chromatin remodelling in liposarcoma tissue samples using a limited panel of histone modifications associated with gene activation and repression. An increased binding of positively acting modifications, acetylation of histone $\mathrm{H} 3$ and histone $\mathrm{H} 4$, was shown in association with higher levels of hTR expression, while no significant differences were shown in association with hTERT expression. In addition, no significant alterations in the binding of the negatively acting modifications, tri-methyl lysine 9 of histone $\mathrm{H} 3$ and trimethyl lysine 20 of histone $\mathrm{H} 4$, at the telomerase gene promoters were associated with either hTR or hTERT expression. The association between histone acetylation and hTR expression has been shown previously in cell line models; however, this is the first time that the correlation between histone modification and gene expression has been demonstrated in clinical samples.

It has been shown that APBs are not always associated with the ALT phenotype (Fasching et al, 2005) and although candidate genes have been suggested (Jiang et al, 2007), no molecular markers for the detection of ALT have yet been confirmed. Previous work in cell line models highlighted an association of trimethyl $\mathrm{K} 20$ of histone $\mathrm{H} 4$ with both telomerase gene promoters in ALT cell lines, suggesting that chromatin modifications may have potential as novel markers of the ALT phenotype (Atkinson et al, 2005). We investigated the potential for our limited panel of chromatin modifications, in association with the telomerase gene promoters, to distinguish among the three phenotype groups. Although no significant differences in chromatin remodelling were found among the three groups, trends of association were shown with acetyl $\mathrm{H} 3$ and $\mathrm{H} 4$ at the hTR promoter. Perhaps by using a more extensive panel of modifications or a genome-wide screen, other candidate molecular markers for the ALT phenotype could be uncovered in future experiments.
While we found no significant differences among the three TMM phenotype groups using the limited panel of chromatin modifications studied, differences were highlighted in telomerase gene expression itself. We further investigated expression of hTR and hTERT in relation to histological subtype, tumour grade and patient survival as potential prognostic indicators in liposarcoma. While hTERT expression was devoid of prognostic significance, statistical analysis highlighted a trend in favour of worse prognosis for patients with tumours expressing high levels of hTR. No significant association was found between hTR abundance and histological subtype or tumour grade when hTR expression was considered as a dichotomous variable; however, a trend in association of high hTR with the more aggressive subtypes and significant association of high hTR with grade 3 tumours was highlighted when median expression levels of hTR were considered. To our knowledge, this is the first time that such an association between hTR expression and poor prognosis for patients with liposarcoma has been documented.

Evidence is accumulating in support of the relationship between telomerase gene expression, TMM and prognosis. It has been shown in a number of mesenchymal tumour types that the presence of any TMM is predictive of a poorer outcome in patients (Ulaner et al, 2003; Sanders et al, 2004; Costa et al, 2006). The statistical analysis of our patient material with relation to TMM showed that patients with tumours expressing the ALT phenotype had the lowest survival rate at follow-up. Furthermore, those patients whose tumours neither expressed telomerase nor ALT had the highest survival rate with a lower HR for death than those expressing telomerase or the ALT phenotype, although this was only suggestive of significance when adjusted for tumour grade. Consistent with previously published results (Costa et al, 2006), the more aggressive histological subtypes, dedifferentiated or normal-myxoid and round-cell myxoid, were found within ALT or $\mathrm{Tel}+$ groups, respectively, while the least aggressive welldifferentiated liposarcomas were all within the ALT-/Tel- group. Similarly, the highest proportion of grade 3 tumours were found within the ALT group and the majority of Tel+ tumours were grade 2, while those that were ALT-/Tel- were mostly of the least aggressive grade 1 . The association of the ALT phenotype with clinical aggressiveness has been shown previously for liposarcoma (Costa et al, 2006); however, the prognostic significance of ALT appears to be tissue and tumour type-specific. In osteosarcoma, hTERT is a predictive indicator of worse prognosis, with a trend in favour of shorter progression-free survival in patients whose tumours expressed telomerase rather than ALT (Sanders et al, 2004), while in glioblastoma multiforme the presence of ALT is actually indicative of a better prognosis (Hakin-Smith et al, 2003). The correlation between TMM, telomerase gene expression and the various tissue-specific clinical outcomes may reflect the underlying complexity of telomere biology in different tumour types and certainly is of great importance to understanding telomere maintenance and telomerase regulation.

As both expression of the ALT phenotype and high levels of hTR were predictive of poor prognosis as individual factors, we also investigated the prognostic significance of hTR expression as a function of ALT presence. While hTR expression held no prognostic significance within the ALT expressing subgroup, there was a trend in favour of a worse prognosis for patients with ALTtumours expressing high levels of hTR.

From the results of our study and consistent with previous results (Costa et al, 2006), the ALT phenotype is a strong predictor of poor prognosis in liposarcoma; however, we have also shown a novel direct association between high levels of telomerase RNA gene expression and poor prognosis. This is irrespective of any particular TMM but has prognostic significance when the group of liposarcoma samples is considered as a whole, with the high hTR expressing group including some ALT, Tel + and ALT-/Telsamples. We are now beginning to gain some insight into the 
mechanisms regulating the ALT phenotype at the molecular level While the search continues for specific markers to define ALT and to predict the clinical outcome in ALT associated tumours more effectively, the data presented here provide a platform to define the relationship between clinical outcome and the underlying biology of telomere maintenance in cancer.

\section{REFERENCES}

Anderson CJ, Hoare SF, Ashcroft M, Bilsland AE, Keith WN (2006) Hypoxic regulation of telomerase gene expression by transcriptional and posttranscriptional mechanisms. Oncogene 25: 61-69

Atkinson SP, Hoare SF, Glasspool RM, Keith WN (2005) Lack of telomerase gene expression in alternative lengthening of telomere cells is associated with chromatin remodeling of the hTR and hTERT gene promoters. Cancer Res 65: 7585-7590

Bilsland AE, Stevenson K, Atkinson S, Kolch W, Keith WN (2006) Transcriptional repression of telomerase RNA gene expression by c-Jun-NH2-kinase and Sp1/Sp3. Cancer Res 66: 1363-1370

Bryan TM, Englezou A, Dalla-Pozza L, Dunham MA, Reddel RR (1997) Evidence for an alternative mechanism for maintaining telomere length in human tumors and tumor-derived cell lines. Nat Med 3: $1271-1274$

Bryan TM, Englezou A, Gupta J, Bacchetti S, Reddel RR (1995) Telomere elongation in immortal human cells without detectable telomerase activity. EMBO J 14: $4240-4248$

Cong YS, Wright WE, Shay JW (2002) Human telomerase and its regulation. Microbiol Mol Biol Rev 66: 407-425

Costa A, Daidone MG, Daprai L, Villa R, Cantu S, Pilotti S, Mariani L, Gronchi A, Henson JD, Reddel RR, Zaffaroni N (2006) Telomere maintenance mechanisms in liposarcomas: association with histologic subtypes and disease progression. Cancer Res 66: 8918-8924

Fasching CL, Bower K, Reddel RR (2005) Telomerase-independent telomere length maintenance in the absence of alternative lengthening of telomeres-associated promyelocytic leukemia bodies. Cancer Res 65: $2722-2729$

Hakin-Smith V, Jellinek DA, Levy D, Carroll T, Teo M, Timperley WR, McKay MJ, Reddel RR, Royds JA (2003) Alternative lengthening of telomeres and survival in patients with glioblastoma multiforme. Lancet 361: $836-838$

Henson JD, Neumann AA, Yeager TR, Reddel RR (2002) Alternative lengthening of telomeres in mammalian cells. Oncogene 21: 598-610

Hoare SF, Bryce LA, Wisman GB, Burns S, Going JJ, van der Zee AG, Keith WN (2001) Lack of telomerase RNA gene hTERC expression in alternative lengthening of telomeres cells is associated with methylation of the hTERC promoter. Cancer Res 61: 27-32

Jiang WQ, Zhong ZH, Henson JD, Reddel RR (2007) Identification of candidate alternative lengthening of telomeres genes by methionine restriction and RNA interference. Oncogene 26: 4635-4647

Johnson JE, Varkonyi RJ, Schwalm J, Cragle R, Klein-Szanto A, Patchefsky A, Cukierman E, von Mehren M, Broccoli D (2005) Multiple mechanisms of telomere maintenance exist in liposarcomas. Clin Cancer Res 11: $5347-5355$

\section{ACKNOWLEDGEMENTS}

This work was supported by Cancer Research UK, European Community grant LSHC-CT-2004-502943 and LSHC-CT-2005018806, Glasgow University and the Italian Association for Cancer Research.

Keith WN, Hoare SF (2004) Detection of telomerase hTERT gene expression and its splice variants by RT-PCR. Methods Mol Med 97: $297-309$

Kim NW, Piatyszek MA, Prowse KR, Harley CB, West MD, Ho PL, Coviello GM, Wright WE, Weinrich SL, Shay JW (1994) Specific association of human telomerase activity with immortal cells and cancer. Science 266: $2011-2015$

Montgomery E, Argani P, Hicks JL, DeMarzo AM, Meeker AK (2004) Telomere lengths of translocation-associated and nontranslocationassociated sarcomas differ dramatically. Am J Pathol 164: 1523-1529

Sandberg AA (2004) Updates on the cytogenetics and molecular genetics of bone and soft tissue tumors: liposarcoma. Cancer Genet Cytogenet 155: $1-24$

Sanders RP, Drissi R, Billups CA, Daw NC, Valentine MB, Dome IS (2004) Telomerase expression predicts unfavorable outcome in osteosarcoma. J Clin Oncol 22: $3790-3797$

Serakinci N, Hoare SF, Kassem M, Atkinson SP, Keith WN (2006) Telomerase promoter reprogramming and interactio with general transcription factors in the human mesenchymal stem cell. Regenerative Med 1: $125-131$

Ulaner GA, Hoffman AR, Otero J, Huang HY, Zhao Z, Mazumdar M, Gorlick R, Meyers P, Healey JH, Ladanyi M (2004) Divergent patterns of telomere maintenance mechanisms among human sarcomas: sharply contrasting prevalence of the alternative lengthening of telomeres mechanism in Ewing's sarcomas and osteosarcomas. Genes Chromosomes Cancer 41: $155-162$

Ulaner GA, Huang HY, Otero J, Zhao Z, Ben-Porat L, Satagopan JM, Gorlick R, Meyers P, Healey JH, Huvos AG, Hoffman AR, Ladanyi M (2003) Absence of a telomere maintenance mechanism as a favorable prognostic factor in patients with osteosarcoma. Cancer Res 63: 1759-1763

Yeager TR, Neumann AA, Englezou A, Huschtscha LI, Noble JR, Reddel RR (1999) Telomerase-negative immortalized human cells contain a novel type of promyelocytic leukemia (PML) body. Cancer Res 59: 4175-4179

Yi X, Shay JW, Wright WE (2001) Quantitation of telomerase components and hTERT mRNA splicing patterns in immortal human cells. Nucleic Acids Res 29: $4818-4825$

Zhao J, Bilsland A, Hoare SF, Keith WN (2003) Involvement of NF-Y and Sp1 binding sequences in basal transcription of the human telomerase RNA gene. FEBS Lett 536: $111-119$

Zhao J, Bilsland A, Jackson K, Keith WN (2005) MDM2 negatively regulates the human telomerase RNA gene promoter. BMC Cancer 5: 6

Zhao JQ, Glasspool RM, Hoare SF, Bilsland A, Szatmari I, Keith WN (2000) Activation of telomerase RNA gene promoter activity by NF-Y, Sp1, and the retinoblastoma protein and repression by Sp3. Neoplasia 2: $531-539$ 